Z Rheumatol 2009 $68: 7-8$

DOI 10.1007/s00393-008-0419-x

๑) Springer Medizin Verlag 2009
B. Manger ${ }^{1} \cdot$ E. Märker-Hermann ${ }^{2}$

${ }^{1}$ Medizinische Klinik III mit Poliklinik, Universitätsklinik Erlangen-Nürnberg

${ }^{2}$ Klinik Innere Medizin IV (Rheumatologie, klin. Immunologie und Nephrologie) , HSK Dr. Horst Schmidt Kliniken GmbH, Wiesbaden

\title{
Rheuma Update
}

\section{Ausgewählte Manuskripte jetzt im Online-Bereich der Zeitschrift für Rheumatologie verfügbar}

\section{Liebe Kolleginnen und Kollegen,}

nicht nur für uns als Rheumatologen ist kontinuierliche Fortbildung ein essenzieller Bestandteil unserer ärztlichen Aufgaben und Pflichten. Sie dient dazu, einmal erworbene Kompetenzen zu erhalten und zu erweitern sowie sich aktuelle Erkenntnisse zu eigen zu machen. Und sie ist eine der wichtigsten Voraussetzungen, um als Arzt bestmöglich diagnostizieren und therapieren zu können.

Dieser Fortbildungsaufgabe umfassend gerecht zu werden, ist in den letzten Jahren immer komplizierter geworden.

Die größte Herausforderung dabei ist vermutlich, sich auf dem aktuellsten medizinischen Stand zu halten. Die Zahl der wissenschaftlichen Veröffentlichungen wächst von Jahr zu Jahr, und das Tempo, in dem innovative Erkenntnisse gewonnen werden, hat sich rasant gesteigert.

\section{- Was heute noch Goldstandard ist, kann im kommenden Jahr überholt sein.}

Ebenso schnell können schon längst als überholt geltende Methoden - unter Umständen nur geringfügig modifiziert - sich morgen schon wieder als wichtiger Therapiebestandteil etablieren.

Hier in allen für die rheumatologische Klinik und Praxis wichtigen Belangen den Überblick zu behalten, wird immer zeitaufwendiger und ist für den einzelnen Arzt kaum noch ausreichend zu bewälti- gen. Fortbildungen gibt es zwar in großer Zahl, häufig wird jedoch primär über den "State-of-the-Art" informiert, und Innovationen werden nur am Rande gestreift. Andere Veranstaltungen hingegen bieten so viel Neues, dass es schwer fällt, die relevanten, für Klinik und Praxis wichtigen Daten herauszufiltern.

Mit unserem Rheuma Update wagten wir in Kooperation mit der Rheumatologischen Fortbildungsakademie 2006 ein Experiment, das genau dies ändern sollte. Schon an der Premiere nahmen über 300 Rheumatologen teil. Im Jahr 2008 zog das Rheuma Update sogar schon fast 500 Teilnehmer nach Wiesbaden. Dieser Erfolg zeigt, dass die Veranstaltung mit ihrem ungewöhnlichen Konzept eine wichtige Ergänzung des bisherigen Fortbildungsangebots in der Rheumatologie ist.

Aufgeteilt in 15 Themenblöcke gibt das Referententeam unseres Rheuma Updates einmal im Jahr an zwei Tagen im März einen konzentrierten Überblick über die aktuellsten Entwicklungen im gesamten Spektrum rheumatologischer Themen. Im Mittelpunkt der Vorträge und Manuskripte stehen die Erkenntnisse aus Arbeiten, die im jeweils vergangenen Jahr in Peer-Review-Journals publiziert wurden. Auch Abstracts von den bedeutenden rheumatologischen Kongressen sind Thema, wenn sie für Klinik und Praxis zu wichtig sind, um eine Publikation in einer Zeitschrift abzuwarten.

Dabei sieht unser Konzept sehr viel mehr vor, als diese Daten nur zusammen- zufassen. Die Referenten beurteilen auch kritisch die Relevanz der aktuellen Daten für die tägliche Praxis. Sie legen Stärken und Schwächen der Arbeiten offen und geben konkrete Empfehlungen zur Umsetzung der Erkenntnisse bei der Versorgung unserer Patienten.

\section{$\checkmark$ Die für Klinik und Praxis wichtigen Daten stehen im Vordergrund}

Herzstück dieses Seminars ist ein umfangreiches Handbuch, in dem sich ausführliche Manuskripte der Referenten zu allen wichtigen Publikationen aus dem vergangenen Jahr finden. Nicht an die zeitlichen Vorgaben eines Vortrags gebunden, geben unsere Referenten in diesen Texten noch ausführlichere Informationen und berücksichtigen zudem weitere ihnen wichtig erschienene Studien aus dem Vorjahr. Selbstverständlich auch hier: kritisch kommentiert, mit einem Fazit für die praktische und klinische Arbeit.

In Kooperation mit dem Springer-Verlag und speziell der Zeitschrift für Rheumatologie stehen für Abonnenten im Online-Bereich dieser Zeitschrift nun drei Handbuchbeiträge von unserem Rheuma Update 2008 zur Verfügung. Es handelt sich um die Beiträge zu Diagnostik und Outcome der rheumatoiden Arthritis (RA), zum systemischen Lupus erythematodes (SLE) und Antiphospholipidsyndrom (APS) sowie zu seltenen rheumatischen Erkrankungen. 
Im Beitrag zur rheumatoiden Arthritis geht Prof. Klaus Krüger aus München u. a. auf seltene Manifestationen und Befunde ein. Er widmet sich ausführlich Studien zum Einfluss von Autoantikörpern, genetischen Faktoren und Biomarkern auf die Prognose der RA und analysiert zahlreiche Arbeiten, in denen kardiovaskuläre Risikofaktoren und Komorbiditäten im Vordergrund stehen.

Das Manuskript „SLE und APS“ wurde von Prof. Christof Specker, Essen, verfasst. Neben wichtigen Abstracts vom ACR-Kongress 2007 in Boston geht er u. a. auf neue Therapieprinzipien und biologische Substanzen ein, die bei der Behandlung des Lupus an Bedeutung gewinnen. Ein eigenes Kapitel nimmt sich der EULAR-Empfehlungen zum Management des SLE an.

Der Beitrag "Seltene Erkrankungen“ von Prof. Bernhard Manger, Erlangen, geht ausführlich auf Epidemiologie, Klinik, Diagnostik und Therapie der Kristallarthropathien ein und behandelt aktuelle Studien zu periodischen Fiebersyndromen, zum adulten M. Still, M. Behçet, zur Amyloidose und Sarkoidose.

Wir denken, dass diese im Jahr 2008 aktuellen Beiträge auch jetzt noch von profundem Interesse für Sie sind und hoffen, dass Sie und Ihre Klinik oder Praxis von dieser Arbeit profitieren. Sollten wir Ihr Interesse nach mehr wecken, würden wir uns selbstverständlich freuen, Sie auf dem diesjährigen Rheuma Update zu begrüßen, das am 13. und 14. März in Wiesbaden stattfindet. Dort bieten wir Ihnen wieder einen konzentrierten Überblick über die für Rheumatologen wichtigsten Studien aus dem Jahr 2008 und versprechen Ihnen eine optimale Fortbildung, um sich in der Rheumatologie „fit zu halten“.

Mit kollegialen Grüßen,

Ihre

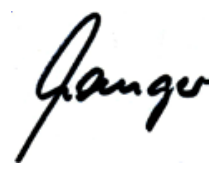

B. Manger<smiles>CC(C)CC(C)C</smiles>

E. Märker-Hermann

\section{Korrespondenzadresse}

Prof. Dr. E. Märker-Hermann

Klinik Innere Medizin IV (Rheumatologie,

klin. Immunologie und Nephrologie),

HSK Dr. Horst Schmidt Kliniken GmbH

Ludwig Erhard Str. 100, 65199 Wiesbaden

elisabeth.maerker-hermann@hsk-wiesbaden.de

\section{Handbuchbeiträge online ...}

Die Handbuchbeiträge des Rheuma Updates 2008 finden Sie online im Volltextarchiv der Zeitschrift für Rheumatologie unter www.ZeitschriftfuerRheumatologie.de im Anhang dieses Editorials
Frühjahrssymposium des Rheumazentrums Württemberg am Sa. 21.03.2009 in Plochingen Aktuelle Konzepte in der Therapie von Kollagenosen

Vorsitz:

W. L. Gross, Lübeck/Bad Bramstedt

R. Maleitzke, Bad Buchau

Programm:

09:30 Uhr Begrüssung

B. Hellmich, Plochingen

09:35 Uhr Systemischer Lupus erythematodes

C. Fiehn, Baden Baden

10:05 Uhr Systemische Sklerose

B. Hellmich, Plochingen

10:35 Uhr Polymyositis/Dermatomyo-

sitis/MCTD

Schnabel, Bad Wildbad

11:05 Uhr Kaffeepause

11:35 Uhr Sjögren Syndrom

T. Dörner, Berlin

12:05 Uhr Stammzelltransplantation bei Kollagenosen

I. Kötter, Tübingen

12:35 Uhr Neues zu ANCA-assoziierten Vaskulitiden

W. L. Gross Lübeck/Bad Bramstedt

13:05 Uhr Schlusswort 